\title{
Dialogue à propos de Carnets de lecture. Généalogie d'une pratique littéraire
}

Daniel Ferrer et Andrei Minzetanu

\section{(2) OpenEdition}

10 Journals

Édition électronique

URL : http://journals.openedition.org/genesis/3779

DOI : 10.4000/genesis.3779

ISSN : 2268-1590

Éditeur :

Presses universitaires de Paris Sorbonne (PUPS), Société internationale de génétique artistique littéraire et scientifique (SIGALES)

Édition imprimée

Date de publication : 12 décembre 2016

Pagination : 219-226

ISBN : 9791023105490

ISSN : $1167-5101$

\section{Référence électronique}

Daniel Ferrer et Andrei Minzetanu, « Dialogue à propos de Carnets de lecture. Généalogie d'une pratique littéraire », Genesis [En ligne], 43 | 2016, mis en ligne le 10 mai 2019, consulté le 15 septembre 2020. URL : http://journals.openedition.org/genesis/3779

Ce document a été généré automatiquement le 15 septembre 2020.

Tous droits réservés 


\title{
Dialogue à propos de Carnets de lecture. Généalogie d'une pratique littéraire
}

\author{
Daniel Ferrer et Andrei Minzetanu
}

1 Daniel Ferrer - Les carnets d'écrivain, qui constituent depuis longtemps un des objets privilégiés de la critique génétique, semblent susciter actuellement des réflexions nouvelles, comme en témoigne par exemple dans ce numéro de Genesis l'article de Sophie Hébert ou encore l'analyse que fait Sabine Teyssonneyre de ses propres carnets de travail. Le livre que vous publiez sur les carnets de lecture se place dans une perspective plus vaste, qui ne relève pas directement de la critique génétique, mais qui a néanmoins beaucoup à voir avec elle et qui lui apporte à mon avis de nombreux éléments de réflexion. Ce travail, qui traverse les époques et les langues avec une impressionnante érudition, soulève bien des problèmes et je vous propose d'en évoquer ensemble quelques-uns.

Andrei Minzetanu - Ce sera avec grand plaisir. Si vous voulez, on peut commencer notre dialogue en partant du texte de Sophie Hébert. J'ai lu attentivement son texte et si je vous propose qu'on le prenne comme point de départ, c'est parce que je considère qu'il présente d'une manière très intéressante les recherches consacrées à cette problématique depuis quelques années et les difficultés liées à une définition du carnet, à son fonctionnement générique, mental, social, génétique et littéraire. Son article, « Du document de genèse à la genèse d'un genre : le carnet d'écrivain dans la littérature française $\mathrm{du} \mathrm{xx}^{\mathrm{e}}$ siècle ", essaie de comprendre «la fabrique d'un genre " à partir de deux dimensions qu'elle considère comme essentielles : l'aspect matériel du carnet et l'utilisation qu'en font les écrivains. Ce choix théorique conduit $\mathrm{S}$. Hébert à une figure de l'écrivain « carnétiste » et à une définition du carnet comme un " genre » détenteur d'une «vision spécifique du monde» et «révélateur d'un style de vie». Je partage l'intérêt pour la matérialité du carnet et pour tout ce que la critique génétique peut nous apprendre sur ses conséquences pratiques. Par contre, je crois que le rôle de cette matérialité ne devrait pas être surestimé et que, dans ce cas particulier, la génétique 
textuelle ne peut pas se contenter de nous renvoyer à l'histoire des supports. Il est indiscutable que cette matérialité représente un conditionnement dans le travail de création - les écrivains eux-mêmes le soulignent souvent - mais son pouvoir explicatif est, me semble-t-il, assez réduit. Je dirais que ce conditionnement matériel ne doit pas cacher des conditionnements autrement plus importants qui revoient, à mon sens, à l'institution scolaire et à l'histoire de la lecture. Pour le dire plus directement, le carnet matériel ne doit pas nous empêcher de voir ce que l'on pourrait appeler le «carnet mental », le carnet que l'écrivain ou le lecteur a dans la tête.

D. F. - Il faut se souvenir que la visée de Sophie Hébert, dans sa thèse, est poétique et surtout générique, et seulement accessoirement génétique (nous lui avons évidemment demandé de déplacer l'accent pour son article dans notre revue). Son but ici est moins d'établir une nomenclature que d'arriver à cerner le genre du carnet. Elle procède par «ressemblance familiale», un faisceau de traits dont aucun n'est commun à tous les membres de la famille.

4 La question (y a-t-il un genre du carnet, comment le définir, comment apparaît-il ?) me paraît intéressante dans la mesure où elle met en évidence un croisement fécond de problèmes génériques et génétiques. Il faut dire que la place des genres dans la genèse a été jusqu'à présent très insuffisamment étudiée. Ici la question se pose de façon particulièrement retorse, puisqu'on semble assister à la naissance d'un genre, au cours du $\mathrm{xx}^{\mathrm{e}}$ siècle, à partir de la publication, de plus en plus fréquente, de ce qui était de purs documents génétiques, mais qui va finir par devenir un modèle d'écriture, un « carnet que l'écrivain ou le lecteur a dans la tête » et donc produire des effets de genèse en retour, notamment à travers ce que vous appelez dans votre livre la posture adoptée par les écrivains.

5 Il est vrai que l'importance de la matérialité du support ne doit pas être surestimée, mais jusqu'ici elle était plutôt sous-estimée. Son pouvoir explicatif est réduit si on la considère isolément et non comme élément d'un faisceau de facteurs. Les écrivains sont-ils conditionnés par les supports existants? Dans une certaine mesure, mais l'existence des supports est aussi conditionnée par la pratique des écrivains : Stendhal coud lui-même ses cahiers ou fait interfolier ses volumes pour créer des supports adéquats à ses besoins; bien des écrivains, adoptant des produits commerciaux, se les approprient en traçant des marges, en délimitant des sections ; si Moleskine offre une telle gamme de carnets, au lieu de standardiser un ou deux modèles, c'est bien qu'il y a des demandes très spécifiques adaptées à des pratiques diverses, d'un écrivain à l'autre et aussi chez un même écrivain. On voit bien que Proust utilise de façon diverse petit agenda, carnets longilignes et cahiers, même s'il y a des recoupements. On peut dire que l'écrivain, le support (et tout le dispositif matériel de travail) et l'écriture sont dans un rapport dialectique de (re)production mutuelle, qui est lui-même conditionné plus ou moins étroitement par un environnement social.

6 En fait je ne sais pas si vous faites bien de distinguer carnet matériel et «carnet mental ». (J'aurais tendance à croire qu'on ignore ce que « l'écrivain a dans la tête » et qu'on ne peut qu'observer des traces qui témoignent d'une suite d'actions "situées ", mais laissons cela pour le moment.) Carnet mental et carnet matériel forment un système indivisible, cet «extended mind " que Dirk Van Hulle met en avant dans son important ouvrage récent ${ }^{1}$.

7 L'institution scolaire, qui propose un cadre préétabli à ce rapport dialectique, a bien sûr une importance capitale, ainsi que l'histoire de la lecture, mais leur pouvoir 
déterminant ne doit pas être surestimé non plus, car on voit bien que des écrivains de même époque travaillent de manières très différentes.

8 A.M. - Avant de revenir sur ces questions, je souhaiterais mieux situer, méthodologiquement parlant, la réflexion que je développe dans Carnets de lecture, généalogie d'une pratique littéraire. La question qui m'a occupé était celle d'un geste particulier de lecture, celui du lecteur qui tombe sur une phrase qui le bouleverse, qui l'intrigue d'une manière ou d'une autre, et qu'il a envie de prélever et de garder dans un carnet spécialement conçu à cet effet. Par conséquent, l'objet précis de cette recherche renvoie à ce geste de lecture et, plus généralement, aux carnets de citations, à l'articulation qui existe entre le carnet de lecture et le carnet de citations.

9 Afin de comprendre ce geste et cette articulation, j'ai préféré à l'approche générique que vous évoquiez tout à l'heure une approche du carnet comme objet et comme pratique; la matérialité du carnet est très importante effectivement; néanmoins, j'ai l'impression que ce qui est déterminant ici n'est pas tellement le conditionnement mental ou pratique du support, mais surtout un changement de regard qui nous a conduits à la prise en considération de l'intégralité du carnet. Les carnets de lecture étaient souvent transformés, par les éditeurs ou par les écrivains eux-mêmes, en recueils d'" essais ", de "pensées » ou d'« aphorismes "; l'avènement, au xx siècle, de ce que vous appelez le « genre du carnet » a rendu sans doute les lecteurs plus sensibles à l'origine de ces notes (de lecture) et à leur contexte.

10 L'objectif de ma recherche a été de comprendre ce geste précis de lecture - le geste de l'extrait - dans un cadre théorique qui inclut un type particulier de lecteur (le lettré "excerpteur») et un régime de lecture que j'ai proposé d'appeler «la lecture citationnelle ${ }^{2} »$. Pour la compréhension de la genèse mentale, historique et littéraire de ce geste, j'ai choisi d'abandonner la piste générique au profit d'une approche qui doit beaucoup à la sociologie culturelle de Pierre Bourdieu, une approche pour laquelle ce geste est avant tout une disposition qui doit être intégrée à son tour dans un habitus et dans un comportement plus ou moins cohérent de lecteur.

11 D. F. - Votre approche me semble légitime et féconde, mais il y a entre nous une différence d'accent : vous vous intéressez au premier chef à la lecture, et à l'écriture seulement dans la mesure où elle est associée à un acte de lecture (et par ailleurs pratiquement sa seule trace observable) tandis que pour le généticien, c'est évidemment l'écriture qui est centrale, la lecture intervenant dans la mesure où elle est une composante de la genèse.

12 Mais nous pouvons négliger cette différence pour les besoins de la discussion. Nous nous intéressons l'un et l'autre à ce que j'appelle les "dispositifs de lecture-écriture ", dont les carnets sont un exemple emblématique, mais qui peuvent être, selon moi, observés beaucoup plus largement, depuis l'élémentaire rature jusqu'au faisceau d'interactions qui se nouent à l'intérieur d'une revue littéraire, ou à travers une batterie de revues rivales. Je vous rejoins aussi en constatant que ces dispositifs s'enchâssent les uns dans les autres et qu'il est donc artificiel (quoique nécessaire) de les étudier séparément. Il est en tout cas nécessaire, en effet, de considérer le contexte dans lequel ils s'inscrivent et l'articulation des différents niveaux.

13 A. M. - Il est vrai que ce qui m'intéresse avant tout c'est la lecture et, d'une manière plus générale, ce dispositif "lecture-écriture " que vous mentionnez également dans la mesure où j'ai pu remarquer, surtout chez Valéry, que la réflexion sur le 
fonctionnement du plaisir de la lecture entraine des modifications dans la pratique et dans la conception de l'écriture littéraire.

Je tiens à dire un mot aussi sur le rapport de ce livre à la critique génétique : ce qui me passionne avant tout, c'est le lien de la critique génétique avec des disciplines voisines : l'histoire et la théorie littéraires, l'histoire de la lecture, la sociologie culturelle, l'histoire des institutions scolaires, bref, avec une épistémologie de la littérature. Il va de soi que j'ai lu dans ce sens, avec beaucoup d'intérêt, l'échange qu'a eu Pierre-Marc de Biasi avec Bourdieu autour de ces questions ${ }^{3}$. J'aime penser que ce livre contribue, ne serait-ce qu'un peu, aux problématiques de la génétique textuelle; et je suis heureux qu'il ait été accueilli, grâce à Anne Herschberg Pierrot et à Jacques Neefs, dans une collection qui s'intitule « Manuscrits modernes ». En tout cas, quant à l'objet précis qui m'intéressait, je crois que le fait d'avoir intégré les problèmes de la critique génétique dans une réflexion de longue durée, mais aussi dans une confrontation avec des instruments théoriques venus d'ailleurs, m'a beaucoup aidé. J'espère avoir mieux éclairé, grâce à un parcours historique qui insiste beaucoup sur des figures emblématiques comme Érasme et Montaigne, sur la survie de la rhétorique et sur l'institutionnalisation de l'art de l'extrait par la pédagogie jésuite, le geste de l'extrait et le carnet de lecture.

D.F. - C'est une démarche qui me semble complémentaire de celle de la critique génétique et c'est à ce titre que nous avons souhaité vous accueillir dans Genesis.

A. M. - J'en suis très heureux. Je crois aussi que cette ouverture est une des meilleures réponses que l'on peut apporter aux « résistances » et aux critiques les plus fréquentes adressées à la critique génétique : une méthode très coûteuse en termes de moyens et d'engagement avec des résultats parfois trop modestes, une certaine fétichisation du texte et des supports, une conception très " personnelle » de l'auteur et de la créativité, enfin un certain "positivisme»; sur ce dernier point, je crois que la génétique textuelle s'est imposée à partir des années quatre-vingt dans un contexte épistémologique, culturel et politique qui était plus "paisible", par rapport à l'effervescence des années structuralistes, et qu'elle a connu des tendances théoriques que l'on retrouve un peu partout dans le champ des sciences humaines de ces annéeslà : en histoire, un "retour à l'archive", comme le montre Roger Chartier dans son beau livre, Au bord de la falaise ${ }^{4}$; en sociologie, un retour à l'expérience et à une sociologie de l'acteur, une mise en valeur de la notion de "résistance ", etc. Si la critique génétique prend au sérieux ces questionnements, je crois que les études et la recherche littéraires auront beaucoup à gagner. Inversement, je crois aussi que la théorie littéraire et surtout la théorie de la lecture doivent s'approcher davantage de la critique génétique. C'est ce que j'essaie de faire dans mon livre, en proposant une théorie de la lecture capable d'intégrer les traces matérielles de la lecture, ces phrases prélevées au cours de la lecture, ces «notes-citations ».

D. F. - Votre perspective sur la critique génétique est juste. Mais on peut aussi raconter la même histoire de différentes façons. Dire que la critique génétique prolonge et incarne la conception dynamique du texte qui était celle du poststructuralisme et dont le besoin s'était fait sentir en réaction à la conception structuraliste. Ou encore que l'accent mis sur les processus de création plutôt que sur l'objet littéraire rejoint une tendance esthétique contemporaine qui se manifeste notamment à travers l'avènement de l'art conceptuel. Ou bien que l'intérêt pour les virtualités de l'écriture plutôt que 
pour leur actualisation dans un texte fait partie d'un grand mouvement vers la dématérialisation.

Une double objection à la possibilité d'une théorie telle que celle que vous évoquez se présente à l'esprit. Les traces conservées, et en tout cas celles qui ont été jusqu'à présent analysées, sont le plus souvent celles de lecteurs-scripteurs remarquables: pour le dire vite de grands esprits qui n'écrivent pas comme tout le monde et dont rien ne prouve qu'ils lisent comme tout le monde. Peut-on en tirer une théorie de la lecture à portée générale ? Peut-être suffira-t-il de mieux explorer les archives et d'étudier les carnets de lecture des gens ordinaires pour répondre à cette objection. Mais plus fondamentalement, peut-on extrapoler à partir du cas très particulier des lectures qui s'écrivent, ou qui laissent des traces palpables et analysables, pour en tirer des conclusions sur le champ considérablement plus vaste des lectures silencieuses à tous égards? Qu'en pensez-vous?

A. M. - J'essaierai de répondre à votre double objection tout à fait légitime. Vous dites que les lecteurs-scripteurs que j'analyse ne lisent pas comme tout le monde. Je suis d'accord et je n'ai pas voulu effacer cette différence entre une "lecture ordinaire", pour le dire très vite, et une « lecture lettrée ». Mais il faut noter que le geste de lecture qui est au cœur de mon livre, le geste de l'extrait, traverse l'ensemble de ces pratiques ; c'est un geste que l'on retrouve dans les carnets des jeunes écoliers qui s'approprient ainsi une partie du patrimoine littéraire et dans les carnets des lecteurs professionnels. L'intérêt de l'étude d'un tel geste vient de là. J'ai essayé de montrer que le prélèvement d'un certain type de phrases, que j'appelle "note-citation ", est à comprendre dans le cadre de la survie moderne d'un art de lecture très ancien, l'ars excerpendi, dans un contexte qui lui est épistémologiquement défavorable à cause du déclin de la rhétorique et surtout parce qu'on privilégie de plus en plus, dans tous les milieux du savoir, l'intégralité du texte, son autorité. Mon livre n'est pas original de ce point de vue ; ce genre de questions a déjà été posé depuis longtemps par une certaine critique des sources qui a essayé de comprendre quel a pu être le rôle de l'école et surtout le rôle des recueils de lieux communs dans la naissance des Essais de Montaigne. Je crois aussi que la sociologie de la lecture, qui commence à s'intéresser aux lecteurs non professionnels, confirme l'importance et la portée de ce geste de l'extrait ${ }^{5}$.

Par conséquent, je ne peux pas dire avec certitude que la théorie de la lecture que je propose soit tout à fait généralisable; mais ce que je peux affirmer c'est que le comportement de certains lecteurs lettrés, surtout quant à ce geste particulier de l'extrait, doit être compris dans la continuité d'un geste scolaire et dans un cadre, historiquement parlant, très complexe. Inventer une catégorie ad-hoc, comme celle de " carnétiste », pour décrire et expliquer le comportement de certains lettrés, me paraît risqué parce qu'une telle catégorie occulte une histoire scolaire, spirituelle, rhétorique et critique très riche et qui mérite d'être connue.

Pour finir, je dirai un mot sur la lecture silencieuse et ses rapports avec les traces que l'on trouve dans les carnets, sur la différence, au fond, entre la « lecture réelle » et une lecture reconstituée par ses traces. Il est vrai que mon livre fait le pari que la lecture, une activité incontestablement très complexe, peut être étudiée, connue et comprise. Je combats un certain mythe de l'idiosyncrasie que beaucoup d'écrivains cultivent. Je crois qu'il ne faut pas les croire sur parole et qu'on n'a aucun argument solide qui nous incite à penser que ce que vous appelez « la lecture silencieuse » soit très différente de ce qu'on arrive à voir dans les carnets de lecture. Au contraire, je crois que les carnets 
de lecture peuvent apporter une confirmation supplémentaire à des théories de la lecture aussi intéressantes et puissantes que celle de Michel Charles.

D. F. - On n'a guère non plus d'argument solide pour affirmer que ces lectures soient homologues. Personnellement, je n'ai pas d'idée sur la question et j'accepte très volontiers votre hypothèse à titre d'hypothèse.

A. M. - Oui, je pense qu'on devrait accepter cette hypothèse à titre d'hypothèse ; cela nous permet d'avancer et il ne faut pas oublier malgré tout qu'aujourd'hui on dispose, en sciences humaines, de multiples instruments pour penser et objectiver ces pratiques : j'ai évoqué la sociologie de Bourdieu, mais je pense aussi au travail très intéressant de Bernard Lahire (à sa définition de la disposition, des plis du social, etc.), à celui de Roger Chartier (sur la notion de "représentation »), aux écrits de Jérôme Meizoz (consacrés à la notion de posture), au livre de Marielle Macé (Façons de lire, manières d'être) et à la théorie des textes possibles de Michel Charles et de ses disciples. Postuler inversement que cette «lecture silencieuse » est inaccessible et que notre seule voie est celle, poétique, des écrivains équivaut, à mon sens, à un terrible renoncement. Cela dit, je crois que les informations que nous fournissent les lettrés à ce sujet, grâce à l'introspection (et je pense notamment à Barthes), sont très précieuses.

D. F. - J'aurais mauvaise grâce à vous le reprocher, car la critique génétique s'intéresse bien, elle aussi, à des lecteurs, sinon toujours lettrés (c'est le cas dans la grande majorité des cas), du moins, par définition, lettrant : des lecteurs qui sont avant tout écrivains. Elle s'est donc penchée depuis longtemps sur ces traces de lecture qui sont pour elle d'une importance primordiale. Mais en dernier ressort, malgré tous ses efforts classificatoires et typologiques, elle y cherche sans doute moins des indices de portée anthropologique ou historique générale que des singularités propres à rendre compte de la singularité de l'œuvre (ou du geste créateur).

A.M. - J'en suis conscient et trouve cette démarche très intéressante. Mais cette singularité m'interroge; je me pose des questions dans ce sens sur la portée pédagogique de la critique génétique. Est-ce que cette singularité, des lecteurs par exemple, doit être décrite pour que l'élève soit capable d'en admirer la force et la grandeur, comme le propose déjà l'herméneutique, ou bien, comme le propose la rhétorique, cette description génétique doit servir à la compréhension du fonctionnement de cette singularité, à une compréhension plus générale et active de la créativité artistique?

D.F. - Le but premier n'étant pas pédagogique, il est légitime de se servir de cette recherche à des fins diverses. Les deux démarches que vous décrivez peuvent être poursuivies avec profit me semble-t-il, même si la deuxième me semble plus intéressante.

A. M. - La deuxième démarche me parait aussi enrichissante. De ce point de vue, je dois dire que j'ai essayé de lire votre beau livre Logiques $d u$ brouillon à travers les recherches de Judith Schlanger, et plus particulièrement à travers son livre L'Invention intellectuelle; ces deux tentatives de modélisation de l'activité créatrice me paraissent vraiment profitables ${ }^{6}$. Même si leur but premier n'est pas pédagogique, je crois que la recherche consacrée à la didactique et, plus généralement, à la "crise " des études littéraires devrait s'en inspirer davantage.

D. F. - Il faut rappeler cette évidence que les singularités ne peuvent s'apprécier que par rapport aux habitudes et les transgressions par rapport à la norme et que l'ignorance 
de celles-ci est source de regrettables méprises. D'où le grand intérêt de votre panorama historique. Pour prendre un exemple frappant, Joyce, auteur hors norme à bien des égards et fort idiosyncrasique dans sa pratique de l'extrait, n'a jamais caché ce qu'il devait à son éducation jésuite soit tout simplement « how to gather, how to order and how to present a given material ».

A. M. - Sur la singularité, je vous suis sans réserve. De là vient, à mon sens, l'intérêt de la littérature comparée et d'une approche historique. Les gestes singuliers des écrivains ne sont plus enfermés dans le cadre d'une œuvre personnelle ou d'une littérature nationale, mais dans un cadre plus dynamique ; j'évoque par exemple dans mon livre la postérité roumaine des Cahiers de Valéry, le devenir-monument de ces cahiers, et le désir de certaines littératures dites périphériques d'explorer les brouillons de leurs écrivains dans une même veine patrimoniale. Dans ce contexte, les cahiers ont été souvent utilisés, je trouve, comme une confirmation du " génie national ».

En fait, mon livre ne fait que réaffirmer une thèse bien connue qui pose que «l'inconscient c'est l'histoire »; dans la version finale du livre ${ }^{7}$ 'ai supprimé les deux chapitres concernant l'Antiquité et le Moyen Âge, mais un des premiers témoignages que l'on possède sur l'art de l'extrait est un texte de Pline le Jeune sur les pratiques de son oncle, Pline l'Ancien; ce texte a une fortune critique incroyable et peut être considéré comme une sorte de matrice pour les discours que les lettrés tiennent sur ce type de lecture. De la même manière, Judith Schlanger a montré à quel point le texte de Poincaré sur la créativité a joué un rôle majeur sur la conception de la créativité au $\mathrm{xx}^{\mathrm{e}}$ siècle. C'est pour cela je crois qu'il faut bien séparer l'analyse d'une pratique de lecture (à travers ses traces observables) et l'analyse des discours ou de la posture des lettrés. L'objectif d'une telle démarche n'est pas du tout, comme on l'a souvent dit, de nier la singularité ou l'originalité de tel ou tel écrivain ; il s'agit plutôt de voir comment cette singularité se construit avec et contre une institution, un champ particulier, etc. De ce point de vue, l'analyse de l'influence de la pédagogie jésuite me paraît très significative. Cette pédagogie peut constituer un très bon repère par rapport auquel on peut mesurer la singularité d'une pratique.

31 D. F. - Il est en effet nécessaire de tenir compte du conditionnement et de l'inculcation scolaire. Mais vous montrez bien que leur effet peut agir à l'inverse (selon les "dispositions ", les "tempéraments ", les "positions dans le champ » et sans doute selon des déterminations individuelles de type psychanalytique...). N'est-il donc pas par conséquent très difficile de mesurer leur efficacité réelle? N'y a-t-il pas danger de surestimer de manière infalsifiable l'action de l'importante tradition que vous avez mise au jour? Corrélativement, pensez-vous qu'il soit possible de réinventer, en toute indépendance, des pratiques de notation très similaires?

A. M. - Sur le conditionnement scolaire, je pense que deux thèses assez différentes peuvent être envisagées : une thèse faible qui pose que la survie moderne de l'art de l'extrait est incompréhensible en dehors de l'histoire des pratiques scolaires de lecture; et une thèse forte qui inclut la première et qui dit, en plus, que cette influence scolaire n'est pas seulement la survie d'un cadre général, une survie culturelle dont il est difficile d'évaluer les conséquences pratiques, mais aussi une contribution à la modification des habitus des lecteurs lettrés étudiés dans le livre à travers les écoles jésuites par exemple. Je dirais donc simplement que mon livre ne démontre que la thèse faible. La thèse forte nécessiterait, j'en conviens volontiers, des études beaucoup 
plus minutieuses. Je dirais donc que l'étude de l'influence scolaire ne doit pas être surestimée mais qu'on a tort lorsqu'on la passe complètement sous silence.

Et enfin, une réponse à votre toute dernière question: Peut-on réinventer, en toute indépendance, des pratiques de lecture similaires? Ma réponse est affirmative; elle repose sur une dimension anthropologique que je mentionne dans le livre, un certain «formulisme» (M. Jousse) : les traditions culturelles se transmettent souvent par des "perles ", par des phrases exemplaires qui condensent bien les valeurs et la culture d'une société donnée; et elle repose aussi sur une vision assez pragmatique du "marché des idées »: je cite à un moment donné un entretien entre Bourdieu et Chartier dans lequel le sociologue dit que nous lisons parce que nous avons un marché d'idées sur lequel nous pouvons réutiliser un certain nombre de phrases, d'idées. Je crois que ces deux dimensions, anthropologique et économique, nous conduisent à penser que deux lecteurs issus de deux milieux culturels très différents peuvent avoir des pratiques de lecture et de notation très similaires tout simplement parce que ces pratiques correspondent à des besoins réels, à un fonctionnement similaire du «marché des idées ${ }^{8} »$.

D. F. - Une autre chose que j'apprécie dans votre réflexion, c'est qu'elle ne laisse pas de côté la question axiologique qui est me semble-t-il cruciale, du moins pour les prochains développements de la critique génétique. Vous abordez, dans le domaine qui vous occupe, ce que j'appellerais le problème de la préférence en évoquant la question : pourquoi retenir telle citation plutôt que telle autre ? Pour y répondre, est-ce que vous n'avez pas tendance à privilégier plutôt une explication " dispositionnelle » : la citation tient sa valeur de ce qu'elle rencontre en moi quelque chose qui l'attendait à mon insu (par exemple une réminiscence), plutôt qu'une explication fonctionnaliste, que la critique génétique, de son côté, a souvent tendance à privilégier : je note ce qui va me servir, dans un but plus ou moins défini. Il est vrai que l'explication dispositionnelle ne peut se passer d'un projet minimal (le projet au moins de noter le notable) et que l'explication fonctionnelle exige la reconnaissance d'une certaine disposition (qui me permet de reconnaître ce qui va me servir).

35 A. M. - Je traite la question de la préférence soit à travers les réflexions psychanalytiques de Barthes, soit à travers la sociologie culturelle de Bourdieu, comme une rencontre heureuse, pour le dire très vite, entre un «champ » et les dispositions singulières d'un " habitus ». Les livres de Judith Schlanger (La lectrice est mortelle, Trop dire ou trop peu : la densité littéraire) évoquent également cette question. Vous avez donc raison quand vous dites que j'ai privilégié la «disposition» à la «fonction». Une explication qui serait uniquement fonctionnelle me paraîtrait trop réductrice. Un écrivain lecteur n'est jamais seulement un écrivain, un individu qui travaille. C'est aussi un sujet, un lecteur qui se laisse emporter. C'est pour cela aussi que j'ai défini la notecitation comme un rapport à soi, comme une "communication de soi à soi", avant d'être un rapport à la mémoire ou à la tradition littéraires. Laurent Jenny, dans sa Vie esthétique, et Marielle Macé dans Façons de lire, manières d'être, ont écrit de très belles pages sur ces questions.

D. F. - Oui, il faut essayer d'aller plus loin que ne le fait Barthes avec l'explication trop vague par le corps, ce corps « qui est dans l'intérieur de la tête », et qui rend compte de toutes les préférences. Pourriez-vous expliciter pour le lecteur de Genesis votre usage des notions d'habitus et de pratique et la manière dont vous les articulez ? 
37 A. M. - Ce sont là, j'en ai pleinement conscience, des notions complexes et assez contestées dans le champ de la sociologie actuelle. Je n'évoquerai pas ici les nombreux reproches qu'on a adressés de toute part (de Raymond Boudon à Nathalie Heinich) à cette notion. Je crois simplement que la notion d'habitus peut être très utile dans l'explication de certains comportements culturels. Et quand je dis « habitus » je pense autant à Panofksy qu'à Bourdieu.

Je prends le mot "pratique » dans son sens habituel précisé par le dictionnaire : "qui concerne l'action, la transformation de la réalité extérieure par la volonté humaine »; " qui détermine la conduite ", " qui concerne le sens des réalités, l'aptitude à s'adapter aux situations concrètes ». Dans mon livre, le mot « pratique » a le sens qu'on lui donne lorsqu'on lit un rapport comme celui d'Olivier Donnat, Les Pratiques culturelles des Français (visite des musées, lectures, expositions, cinéma, etc.).

39 Je m'intéresse donc à une pratique particulière de lecture, l'art de l'extrait, et à sa généalogie culturelle. Mais, comme je l'ai déjà dit, dans le cas des lecteurs professionnels, que j'appelle des «lettrés", il faut essayer de comprendre cette pratique de la lecture et le geste de l'extrait dans un ensemble plus vaste qu'on peut appeler un «habitus», c'est-à-dire un "principe générateur des pratiques intellectuelles ${ }^{9} »$. Bourdieu expliquait les pratiques des sujets comme le produit d'une dialectique complexe entre une situation donnée et un habitus, "un système de dispositions qui fonctionne à chaque moment comme une matrice de perceptions, d'appréciations et d'actions ${ }^{10}$ ».

Les débats théoriques concernant la notion d'habitus se réfèrent, en dernière analyse, à la liberté humaine puisque l'opposition concerne l'agent et l'acteur. On peut transposer ce débat dans le domaine de la lecture et se demander si, lorsqu'un lettré lit, il est plutôt déterminé ou plutôt un acteur libre et conscient, avec ses stratégies, etc. Je ne crois pas qu'il y ait de réponse absolue à cette question. Je dirais simplement que l'idiosyncrasie totale, soulignée souvent par les écrivains, est un mythe. Quand on entre vraiment dans l'intimité mentale d'un lecteur et dans une compréhension profonde du mécanisme de sa lecture, on peut même s'amuser à anticiper ce qu'il va retenir d'une lecture. Cioran par exemple est très prévisible de ce point de vue.

41 D. F. - Attention à ce que Pierre-Marc de Biasi a appelé la " paranoïa » qui guette ceux qui pénètrent dans l'intimité des écrivains par l'intermédiaire de leurs manuscrits et qui les conduit à s'identifier à eux, ou du moins à leur processus d'écriture (ou de lecture $)^{11}$. Je peux vous dire que je croyais connaître l'usus legendi de Joyce au point d'être capable d'anticiper ses notations, mais quand j'en ai fait l'expérience réelle, je me trompais à chaque fois...

42 A. M. - Je suis d'accord; il faut faire attention à ce genre de "paranoïa » et, plus généralement, à tout système explicatif trop hégémonique. Ce que je voulais simplement dire, c'est qu'on a des arguments solides pour éviter un trop grand relativisme et pour penser que, souvent, notre compréhension du comportement des lecteurs est assez profonde. Notre capacité d'anticipation et de prédiction est bien évidemment relative, mais elle n'est pas nulle. Si elle ne se vérifie pas toujours, cela ne veut pas forcément dire que notre système explicatif est faux; cela peut vouloir dire que les paramètres qui entrent en ligne de compte lorsqu'un lecteur décide de prélever ou non une phrase sont vraiment d'une très grande complexité. Prenons par exemple le cas des Cahiers de Cioran. L'analyse des phrases prélevées par Cioran m'incite à penser qu'on devrait séparer deux niveaux : un premier niveau plus abstrait dans lequel telle 
ou telle phrase serait une phrase que le lecteur Cioran pourrait prélever (le conditionnel indique ici un modèle probabiliste) parce qu'elle correspond à son « style » de lecture, à ses préférences confirmées par toute son œuvre, par les centaines de phrases recopiées dans ses cahiers et qui respectent presque toujours le même schéma, etc.; et un deuxième niveau, plus concret, qui analyse, dans une situation donnée, ce que Cioran a vraiment prélevé, en fonction de l'intérêt du moment, de ses disponibilités mentales et spirituelles, etc. Ici, l'analyse n'est plus probabiliste; elle essaie de reconstituer un moment particulier de lecture. Le fait de séparer ces deux niveaux renvoie pour moi à l'idée que l'analyse du comportement d'un lecteur ne peut pas se réduire à ces traces visibles et qu'on doit aussi essayer de comprendre ce qui se passe virtuellement dans sa tête ${ }^{12}$.

D.F. - Les traces renvoient bien entendu à des états mentaux, et seraient incompréhensibles si on les occultait, mais la critique génétique préfère quant à elle décrire des suites d'" actions situées » et comprendre leurs logiques en faisant le moins possible appel à la psychologie. C'est peut-être ici que nos démarches divergent. Cela ne doit pas occulter tout ce qu'elles ont en commun.

\section{NOTES}

1. Dirk Van Hulle, Modern Manuscripts: the Extended Mind and Creative Undoing, from Darwin to Beckett and Beyond, London/Bloomsbury, 2013. Voir le compte rendu par Roger Lüdeke dans Genesis, $\mathrm{n}^{\circ} 42,2016$.

2. «La lecture citationnelle ou l'ars legendi comme ars excerpendi », Littérature, n 168 , décembre 2012, p. 31-42.

3. Pierre-Marc de Biasi, «Le sociologue et le généticien. Suivi d'un entretien avec Pierre Bourdieu », dans Jean-Pierre Martin (dir.), Bourdieu et la littérature, Nantes, Éditions Cécile Defaut, 2010, p. 245-290.

4. Roger Chartier, Au bord de la falaise : l'histoire entre certitudes et inquiétude, Paris, Albin Michel, 2009.

5. Voir G. Mauger, C. Poliak et B. Pudal, Histoires de lecteurs, Paris, Nathan, 1999.

6. Daniel Ferrer, Logiques du brouillon: modèles pour une critique génétique, Paris, Seuil, coll. « Poétique », 2011 ; Judith Schlanger, L'Invention intellectuelle, Paris, Fayard, 1983.

7. Issu d'une thèse de littérature comparée soutenue, sous la direction de William Marx, en 2013, à l'université Paris Ouest Nanterre La Défense.

8. Andrei Minzetanu, « Le marché des idées », Critique, no 821, octobre 2015, p. 775-786.

9. Voir Louis Pinto, Pierre Bourdieu et la théorie du monde social, Paris, Albin Michel, 2002, p. 50.

10. Pierre Bourdieu, Esquisse d'une théorie de la pratique, Genève/Paris, Droz, 1972, p. 178.

11. "Paranoïa-genèse. Remarques sur l'identité des recherches en génétique textuelle ", dans Leçons d'écriture, ce que disent les manuscrits, textes réunis par A. Grésillon et M. Werner, Paris, Minard, coll. « Lettres Modernes », 1985, p. 259-275.

12. Voir l'article de Michel Charles, «Le sens du détail », Poétique, nº 116, 1998, p. 387-424. 


\section{AUTEURS}

\section{DANIEL FERRER}

DANIEL FERRER (ITEM) est auteur, entre autres, de Logiques du brouillon. Modèles pour une critique génétique, Paris, Éditions du Seuil, coll. « Poétique », 2011.

\section{ANDREI MINZETANU}

ANDREI MINZETANU (EHESS) est auteur, entre autres, de Carnets de lecture. Généalogie d'une pratique littéraire, Saint-Denis, Presses universitaires de Vincennes, coll. « Manuscrits modernes », 2016. 